\title{
Deutscher Rheumatologiekongress 2022 \\ vom 31. August bis 3. September 2022, Estrel Congress Center in Berlin
}

Liebe Kolleginnen und Kollegen,

die Rheumatologie ist spannend und lehrreich, unsere Fälle sind garniert mit exzeptionellen Diagnosen und besonderen Verläufen. Wir alle betreuen Patienten, deren Diagnose oder Verlauf durch ihre Seltenheit, die besondere Präsentation des Krankheitsfalles, ihren überraschenden Ausgang oder ihre innovative Therapieform besticht. Dieser Tatsache Rechnung tragend will das Programmkomitee der DGRh für den kommenden Kongress in Berlin das Forum „Der besondere Fall“ fortführen, das in den letzten Jahren großen Anklang gefunden hat.

Im Forum „Der besondere Fall“ sollen Fälle aus dem vielfältigen Repertoire rheumatologischer Krankheitsbilder mit o. g. Besonderheiten präsentiert werden.

Hiermit laden wir Sie ein, uns Ihre außergewöhnlichen Fälle in Abstract-Form zu beschreiben und einzureichen. Die Abstracts sollten gegliedert sein in:

1. Vorgeschichte

2. Leitsymptome bei Krankheitsmanifestation

3. Diagnostik

4. Therapie

5. weiterer Verlauf

Im Titel soll die letztendliche Diagnose nicht genannt sein, um die Spannung zu erhalten.

Die Fälle können im Rahmen des Abstract-Verfahrens der DGRh bis 31. März 2022 online eingereicht werden - mit dem „Call for Abstracts“ informieren wir Sie über die Details.
Wir sind gespannt auf Ihre Beiträge und freuen uns auf eine neue und interessante Session!

Mit freundlichen Grüßen

A. Monse

Prof. Dr. Andreas Krause

Kongresspräsident 2022 der DGRh

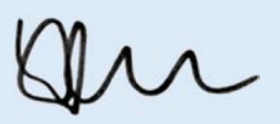

Prof. Dr. Andreas Niemeier

Kongresspräsident 2022 der DGORh

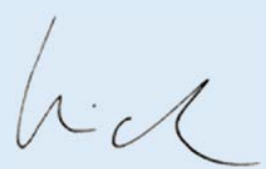

Prof. Dr. Kirsten Minden

Kongresspräsidentin 2022 der GKJR 